\title{
Microvascular involvement in systemic sclerosis: laser Doppler evaluation of reactivity to acetylcholine and sodium nitroprusside by iontophoresis
}

\author{
L La Civita, M Rossi, G Vagheggini, F A A Storino, L Credidio, G Pasero, C Giusti,
} C Ferri

\begin{abstract}
Objectives-To investigate the skin vasodilatory response to iontophoretically applied acetylcholine (Ach), an endothelium dependent vasodilator, and to sodium nitroprusside (SNP), an endothelium independent vasodilator, in patients with systemic sclerosis (SSc).

Methods-Eleven SSc patients were preliminarily studied (10 females, mean age 40.5; mean disease duration 6.5 years), and 16 age and sex matched control subjects. By means of laser Doppler flowmetry skin blood flow was evaluated at third finger, at baseline, and after postischaemic hyperaemia test and during iontophoretically transcutaneous application of $1 \%$ solution of Ach and SNP.

Results-No significant differences in basal skin blood flow were detected between SSc patients and controls. Cutaneous vasodilatory response to ischaemia, Ach, and SNP was significantly less pronounced in SSc patients compared with controls $(p<0.001)$. Moreover, among SSc patients a lower $(p<0.05)$ vasodilatory response to Ach compared with ischaemia and SNP was recorded.

Conclusions-These data confirm a reduction of skin digital vasodilatory reserve in SSc patients and suggest a defect of both endothelial dependent arteriolar relaxation and wall compliance in the pathogenesis of this dysfunction.

(Ann Rheum Dis 1998;57:52-55)
\end{abstract}

Systemic sclerosis (SSc) is a connective tissue disease characterised by diffuse microvasculature changes and progressive fibrosis of the skin and visceral organs, chiefly, lung, heart, gastrointestinal tract, and kidney. ${ }^{1}$ The aetiopathogenesis of SSc is still unknown. However, various pathogenetic hypotheses have been suggested; they include a collagen overproduction by fibroblasts and/or endothelial abnormalities, and/or immunological changes. ${ }^{1-3}$ The microcirculatory abnormalities in SSc patients could be caused by the endothelial dysfunction/damage as suggested by the following clinicoserological manifestations: Raynaud's phenomenon, often preceding the SSc onset, in over $90 \%$ of patients, ischaemic skin lesions, nailfold capillaroscopic abnormalities, increased plasma endothelin-1 and tissue plas- minogen activator, low concentrations of nitric oxide (NO) ${ }^{4-10}$ In addition, an impairment of arteriolar wall compliance has been demonstrated by a reduced post-ischaemic hyperaemia in the digital skin at laser Doppler flowmetry in SSc patients. ${ }^{11}$ However, the pathogenetic relevance of microvascular abnormalities in the SSc still remains to be defined.

The recent use of iontophoresis, a noninvasive method able to deliver in the skin various deionised drugs, including some vasodilators, has permitted a more accurate assessment by laser Doppler flowmetry of the microvascular endothelial cell function in normal and pathological conditions. ${ }^{12-16}$

To verify the role of both endothelial cell dysfunction and reduced microvascular compliance in the scleroderma microangiopathy, we preliminarily investigated in SSc patients the response of skin microvasculature to the iontophoretic application of acetylcholine (Ach), an endothelium dependent vasodilator, and sodium nitroprusside (SNP), an endothelium independent vasodilator, compared with the vasodilatory effect resulting from ischaemia.

\section{Methods}

PATIENTS

Eleven consecutive SSc outpatients (10 females; mean (SD) age 40.5 (10.4), range 21-57; disease duration 6.5 (5.8) years, range 1-18) followed up at the Rheumatology Unit of the University of Pisa, and 16 healthy control subjects, age and sex matched, were enrolled for the study. Patients with SSc were classified according to the American Rheumatism Association Subcommittee for Scleroderma Criteria. ${ }^{17}$ In addition, patients were divided into three cutaneous subsets on the basis of the extent of skin sclerosis, namely limited, intermediate, and diffuse Ssc. ${ }^{518}$ Exclusion criteria were: age $\geqslant 60$ years, smokers, presence of heart and renal failure, severe digital skin ulcers, arterial hypertension, diabetes mellitus, and any other disease possibly responsible for microvasculopathy; moreover, people taking vasodilator or anti-inflammatory treatment were studied after a two week washout period. Patients were asked not to consume caffeine containing drinks during the 24 hours preceding the study.

All subjects gave their informed consent before entering in the trial, which was approved by our Institutional Ethical Committee. 


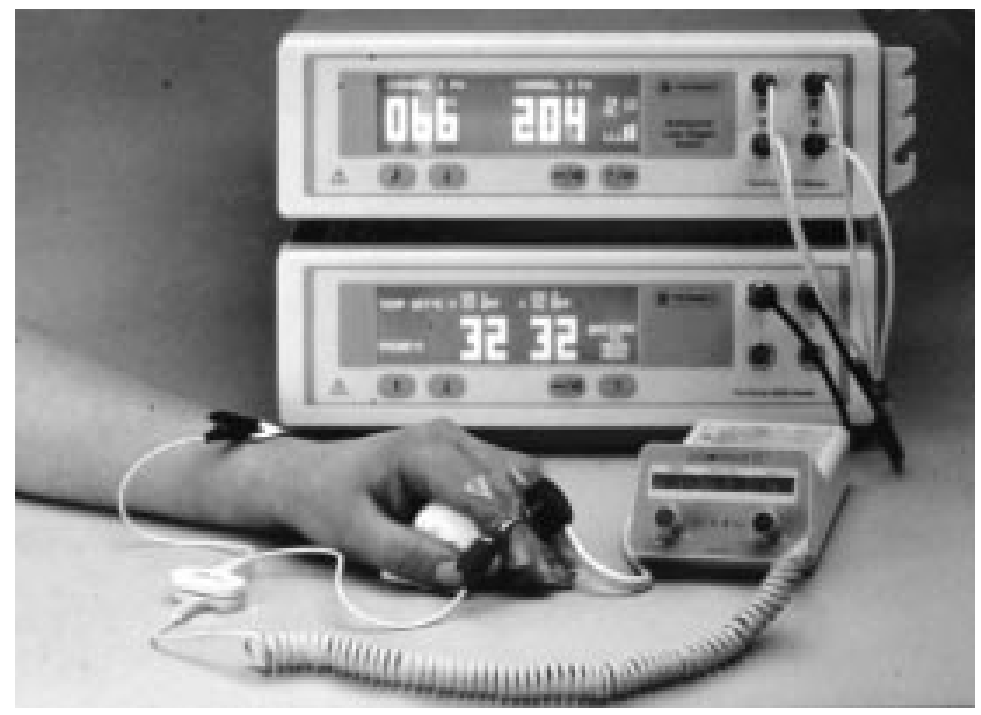

Figure 1 Apparatus of the laser Doppler iontophoresis.

At the entry, a careful clinicoserological assessment was carried out in all patients, as previously described. ${ }^{56}$ Nailfold capillary examination was performed using a Leitz microscope and interpreted according to the criteria of Maricq et al. ${ }^{19}$

MICROCIRCULATORY ASSESSMENT

The skin blood flow evaluation was carried out on the patient in seated position after 30 minutes of acclimatisation at a temperature of $22 \pm 1^{\circ} \mathrm{C}$. Digital blood flow was measured by a laser Doppler flowmeter (Periflux PF4001, standard probe PF408, Perimed, Sweden). The laser Doppler apparatus (fig 1) was connected via RS232 interface with a PC Notebook (Zenith Data Systems, Taiwan), which analysed on line the information on the skin blood flow by a Perisoft program.

POST-ISCHAEMIC HYPERAEMIA TEST

The laser probe was placed on the dorsal surface of the right third finger by a double sided adhesive disc. After the recording of digital basal blood flow for five minutes, a microcuff was applied around the finger and inflated up to $250 \mathrm{~mm} \mathrm{Hg}$ for a three minute period; then, it was instantaneously deflated and digital blood flow was again recorded until the flow was comparable with the baseline one. The value of basal digital blood flow was expressed as conventional perfusion units (PU), while post-ischemic blood flow as "area under curve" (PU in function of the time) recorded during the last two minutes preceding and at the end of ischaemia, respectively. The ratio of these values expressed in percentages indicated the vasodilatory response to ischaemia.

IONTOPHORETIC STUDY

After a five hour interval from the postischaemic hyperaemia test, patients underwent iontophoresis procedure (Periont, Perimed, Sweden) to assess their vasodilatory response to Ach (acetylcholine $\mathrm{HCl}$, Farmigea, Pisa, Italy) and SNP (sodium nitroprusside, $\mathrm{Ma}$ lesci, Milan, Italy) application. Before the ion-
Table 1 Clinicoserological features of 11 systemic sclerosis (SSc) patients

\begin{tabular}{ll}
\hline & $\%$ \\
\hline Female-male & $10-1$ \\
Age, mean (SD) (range) (y) & $40.5(10.4)(21-57)$ \\
SSc duration, mean (SD) (range) (y) & $6.5(5.8)(1-18)$ \\
SSc subsets & 36 \\
Limited & 18 \\
Intermediate & 46 \\
Diffuse & 100 \\
Raynauld's phenomenon & 100 \\
Telangectasias & 72 \\
Skin digital pitting & 18 \\
Cutaneous ulcers & 18 \\
Calcinosis & 72 \\
Oesophageal dysmotility & 91 \\
Lung fibrosis & 27 \\
Pulmonary hypertension & 55 \\
Heart involvement & \\
Autoantibodies & 82 \\
Anti-nuclear & 46 \\
Anti-ENA & 9 \\
Anti-centromere & 72 \\
Anti-Scl70 & \\
\hline
\end{tabular}

*Anti-ENA: anti-extractable nuclear antigen; anti-Scl70: antitopoisomerase I antibody.

tophoretic application, the above drugs were freshly diluted at the concentration of $1 \%$ in sterile deionised water. By means of a silver chloride electrode, supporting a sponge (12 $\mathrm{mm}$ in diameter) of an absolutely ion free material, with a high absorption and without any effects on the drug, 100 microlitre of Ach and SNP were used for the test. Both drugs were randomly introduced, with an interval of two hours, into the cutaneous tissue using an anodal (for Ach) and cathodal (for SNP) charge $\left(0.30 \mathrm{~m}^{\circ} \mathrm{A}\right)$ for 20,30 , and 40 seconds, at constant time intervals of 180 seconds. Through a pocket sited in the middle of drug electrode a small laser diode lighted a limited area of digital skin. The "drug electrode" was fixed to a thermostated probe allowing a constant temperature of $36^{\circ} \mathrm{C}$.

Both basal and maximum blood flow during the Ach and SNP application were respectively expressed as the value of the "area under curve", measured two minutes before the iontophoresis and during the first two minutes subsequent to each application. The per cent of increment of such values defined the maximum vasodilatory response to each drug.

The intra-subject reproducibility of this protocol has been evaluated in six healthy volunteers. The coefficient of variation of the maximum vasodilatory response to Ach and SNP was $49 \pm 21$ and $27 \pm 14 \%$, respectively. Day to day reproducibility to the same substances, determined in four subjects on three occasions, was $36 \pm 17$ and $21 \pm 13 \%$, respectively.

\section{DATA ANALYSIS}

The basal skin blood flow, the maximum vasodilatory response to ischaemia, Ach, and SNP were compared between SSc patients and control subjects by Student's $t$ test. In each group the variability in response to Ach and SNP stimulation was assessed using two way analysis of variance for repeated measures (ANOVA). In both tested groups, the maximum vasodilatory response to Ach and SNP application were compared by using Student's $t$ test for two tails. 
Table 2 Per cent increment of maximum vasodilatory response (area under curve) to ischaemia, acetylcholine (Ach), and sodium nitroprusside (SNP) in SSc patients and control subjects

\begin{tabular}{llllll}
\hline & Ischaemia & p Value & Ach & p Value & SNP \\
\hline SSc patients (11) & $119.9(73.1)$ & 0.05 & $49.2(41.8)$ & 0.05 & $112(82.3)$ \\
p Value & 0.001 & NS & 0.001 & NS & $528(172.2)$ \\
Controls (16) & $461.6(168.3)$ & $454.2(361.4)$ & N & \\
\hline
\end{tabular}

Data shown as mean $(\mathrm{SD})$.

The results are expressed as mean $(\mathrm{SD}) ; \mathrm{p}$ values $<0.05$ were considered significant.

\section{Results}

Table 1 summarises the main epidemiological and clinicoserological findings of $11 \mathrm{SSc}$ patients. In all subjects Raynaud's phenomenon was present at the time of the diagnosis of $\mathrm{SSc}$; in seven cases this manifestation preceded, from 1 to 15 years, the clinical onset of the disease. Other microvascular complications, including teleangectasias (100\%), fingertip pitting lesions $(72 \%)$, and cutaneous ulcers on the lower limbs $(18 \%)$, were also recorded. Among visceral organ involvement interstitial lung fibrosis was demonstrated in most patients. In all cases nailfold capillary microscopy examination showed a typical scleroderma pattern - that is, reduced capillary number and severely enlarged loops (table 1 ).

At baseline, skin blood flow did not show any significant difference between SSc patients (16.3 (6.7) PU) and controls (21.8 (12.3) PU); however, one patient with cutaneous ulcers on the legs showed a basal blood flow considerably lower than the mean value.

After the post-ischaemic hyperaemia test the per cent increment of vasodilatory response was significantly lower in SSc patients than in controls (119 (73.1\%) compared with 461.6 (168.3)\%; p<0.001) (table 2).

The maximum vasodilatory effect to Ach and SNP stimulation was recorded at the third application of the drug in both SSc patients and controls. However, a significant dose related increment in vasodilatory response to the drugs was detected only in controls ( $p$ $<0.001$, ANOVA test). In SSc patients a significant change in maximum vasodilatory effect was recorded when compared with controls $(\mathrm{p}<0.001$; table 2$)$. With regard to the maximum vasodilatory response to the different stimuli (ischaemia, Ach, and SNP), SSc patients showed a lower response to Ach $(p<0.05$, table 2) than SNP and ischaemia. In contrast, no significant variations were seen in control subjects.

In SSc patients no correlations were observed between the microvascular involvement and different cutaneous subsets or capillaroscopic abnormalities.

\section{Discussion}

This study represents the first attempt to use the iontophoretic technique together with laser Doppler flowmetry to evaluate the digital microcirculatory involvement in SSc patients. Although the pathogenesis of scleroderma microangiopathy is still uncertain, abnormally high values of endothelin-1 and reduced plasma concentrations of NO in SSc patients suggest a dysfunction or damage to the endothelium, or both. ${ }^{5-10}$ On the other hand, some investigations with laser Doppler flowmetry have shown a reduced vasodilatory response to ischaemia stimulation in cutaneous microvasculature of SSc patients, which may reflect structural changes in digital arteriolar walls associated with a low vessel compliance. ${ }^{1120}$

Given the above findings we preliminarily investigated the microvasculature in a limited number of SSc patients by using two different methodologies - that is, the post-ischaemic hyperaemia test and iontophoresis. The second method is a well known non-invasive technique that allows the introduction through the skin, by means of a small electric current, of different ionised substances. ${ }^{12}$

Iontophoresis, initially used for local application of therapeutical agents, ${ }^{21}$ is now available to test the effects of some vasodilatory agents on cutaneous microvasculature dysfunction resulting from endothelial cell or muscle wall injury, or both. ${ }^{13-16}$

According to previous findings, ${ }^{11}{ }^{20}$ our study confirms that SSc patients show a reduced vasodilatory reserve of the skin microvasculature in response to ischaemia. Moreover, the vasodilatation by iontophoretically Ach and SNP application is considerably impaired in SSc patients compared with age and sex matched controls. On the basis of these findings, we could hypothesise that digital cutaneous sclerosis reduces the iontophoretic transfer of vasoactive agents across the skin. However, after different stimuli (Ach, SNP, and ischaemia) SSc patients showed a variable vasodilatory response; in fact, the maximum blood flow caused by Ach was significantly lower than that seen with SNP and ischaemia. It is possible to hypothesise that the reduction in vasodilatory response to Ach and SNP could be related to a defective absorption of two drugs because of the cutaneous fibrosis in SSc patients. The vasodilatation secondary to SNP did not show a difference compared with hyperaemia after the ischaemia test, which represents the maximum stimulus for vasodilatation, in both control subjects and SSc patients; this suggests an adequate iontophoretical diffusion of these drugs across the normal skin and the absence of a "railing effect" of cutaneous sclerosis. In our SSc patients a low vasodilatory response caused by $\mathrm{SNP}$ and ischaemia might reflect structural or functional abnormalities of the smooth muscle of arteriolar wall. However, the lower vasodilatory effect related to Ach, compared with SNP, suggests a further dysfunction of endothelial cells, as previously hypothesised. ${ }^{67910}$ 
In contrast, Anderson et al ${ }^{16}$ did not find, on the forearm, different vasodilatory responses to Ach and SNP iontophoresis between Raynaud's, SSc patients, and control subjects. These contrasting results might be explained by the choice of different sites for the iontophoretic study; the microvascular involvement is significantly more pronounced in the digital district.

On the whole, these preliminary findings further support the pathogenetic role of both endothelial and arteriolar wall dysfunction in the microangiopathy of SSc patients. These abnormalities can be evaluated by means of iontophoresis, a non-invasive and easily reliable method. Further studies on a larger patient population could better define the relative role of endothelial and arteriolar wall dysfunction on the severity of organ damage at different stages of the disease.

1 Medsger TA jr. Systemic sclerosis (scleroderma), eosinophilic fasciitis, and calcinosis. 11 th ed. In: McCarty DJ,
ed. Arthritis and allied conditions. Philadelphia: Lea \& Febiger, 1989:1118-65.

2 Korn JH. Immunologic aspects of scleroderma. Curr Opin Rheumatol 1991;3:947-52.

3 White B. Immunopathogenesis of systemic sclerosis. Rheum Dis Clin North Am 1996;22:695-708.

4 Kahaleh MB. The vascular endothelium in scleroderma. Int Rev Immunol 1995;12:227-45.

5 Ferri C, Bernini L, Cecchetti R, Neri R, Latorraca A, Marotta G, et al. Cutaneous and serological subsets of sysMarotta G, et al. Cutaneous and serological subse

6 Ferri C, La Civita L, Zignego AL, Longombardo G, Del Chicca MG, Pedrinelli R, et al. Plasma levels of endothelin-1 in mixed cryoglobulinaemia patients. $\mathrm{Br} J$ Rheumatol 1994;3

7 Yamane K, Miyauchi T, Suzuki N, Yuhara T, Akama T, Suzuki $\mathrm{H}$, et al. Significance of plasma endothelin-1 levels in patients with systemic sclerosis. J Rheumatol 1992;19: 1566-71.
8 La Civita L, Giuggioli D, Del Chicca MG, Longombardo G, Pasero G, Ferri C. Effect of isradipine on endothelin-1 plasma concentrations in patients with Raynaud's phenomenon. Ann Rheum Dis 1996;55:331-2.

9 Godin-Ostro E, Mitrane M, Heller I, Kim H, Seibold JR. Plasma plasminogen activator in systemic sclerosis. [Abstract]. Arthritis Rheum 1985;28 (4/S):80.

10 Silver RM: Scleroderma. Clinical problems. The lungs. Rheum Dis Clin North Am 1996;22:825-40.

11 Wigley FM, Wise RA, Mikdashi J, Schaefer S, Spence RJ. The post-occlusive hyperemic response in patients with systemic sclerosis. Arthritis Rheum 1990;33:1620-5.

12 Morris SJ, Shore AC. Skin blood flow resoponses to the iontophoresis of acetylcholine and sodium nitroprusside in man: possible mechanisms. J Physiol (London) 1996;462: 531-42.

13 Lindblad LE, Ekenvall L, Etzell BM, Bevegard S. Adrenoceptors in Raynaud's disease. J Cardiovasc Pharmacol 1989;14:881-5.

14 Morris SJ, Shore AC, Tooke JE. Responses of the skin microcirculation to acetylcholine and sodium nitroprusside in patients with NIDDM. Diabetologia 1995; 38:1337-44.

15 Morris SJ, Shore AC, Tooke JE. Impaired skin microvascular endothelial cell function in subjects with fasting hyperglycaemia. Int J Microcirc Clin Exp 1996;16 (suppl 1): 284 .

16 Anderson ME, Hollis S, Moore T, Jaison MIV, Herrick AL. Non-invasive assessment of vascular reactivity in forearm skin of patients with primary Raynaud's phenomenon and systemic sclerosis. Br J Rheumatol 1996;35:1281-8.

17 Subcommittee for Scleroderma Criteria of the American Rheumatism Association Diagnostic and Therapeutic Criteria Committee: Preliminary criteria for the classification of systemic sclerosis (scleroderma). Arthritis Rheum 1980; 23:581-90.

18 Masi AT. Classification of systemic sclerosis (scleroderma): relationship of cutaneous subgroups in early disease to outcome and serological reactivity. J Rheumatol 1988;15: 894-8.

19 Maricq HR. Widefield capillary microscopy: technique and rating scale for abnormalities seen in scleroderma and related disorders. Arthritis Rheum 1981;24:1159-65.

20 Goodfield M, Hume A, Rowell N. Reactive hyperemic responses in systemic sclerosis patients and healthy controls. J Invest Dermatol 1989;93:368-71.

21 Garagiola U, Dacatra U, Braconaro F, Porretti E, Pisetti A, Azzolini V. Iontophoretic administration of pirprofen or lysine soluble aspirin in the treatment of rheumatic diseases. Clin Ther 1988;10:553-8. 